\title{
Current correlators in the coordinate space at short distances
}

JLQCD Collaboration:

Masaaki Tomii*

Physics Department, Columbia University, New York 10027, USA

E-mail: mt3164declumbia.edu

\section{Hidenori Fukaya}

Department of Physics, Osaka University, Toyonaka 560-0043, Japan

\section{Shoji Hashimoto and Takashi Kaneko}

KEK Theory Center, Institute of Particle and Nuclear Studies, High Energy Accelerator Organization (KEK), Tsukuba 305-0801, Japan

Department of Particle and Nuclear Physics, SOKENDAI (The Graduate University for Advanced Studies), Tsukuba 305-0801, Japan

\begin{abstract}
We calculate the vector and axial-vector current correlators in the coordinate space and compare them with the experimental information obtained through the spectral functions of hadronic $\tau$ decays measured by ALEPH. Lattice data are obtained with 2+1 Möbius domain-wall fermions at three lattice spacings $0.044,0.055$ and $0.080 \mathrm{fm}$ and the continuum limit is taken. The correlators calculated on the lattice after extrapolating to the physical point agree with those converted from the ALEPH data.
\end{abstract}

34th annual International Symposium on Lattice Field Theory

24-30 July 2016

University of Southampton, UK

\footnotetext{
* Speaker.
} 


\section{Introduction}

Current correlators provide a rich source of information on the QCD vacuum. Their characteristics vary depending on the distance between the currents. Correlators at short distances behave perturbatively providing the information on the strong coupling constant, while those at long distances are saturated by the ground state reflecting the individual mass spectrum and decay constant. In the middle distances between perturbative and non-perturbative regimes, on the other hand, perturbative approaches and low-energy effective theories are no longer suitable to analyze correlators.

Lattice calculation is useful to calculate current correlators at any distances. So far, lattice calculation of current correlators has been aimed mainly at long distances to extract hadron masses and decay constants and shown the agreement with experiments. This agreement supports the consistency between experiments and QCD at low energies, where contributions of excited states are missing and only a part of QCD can be seen. Comparison of lattice correlators at short and middle distances with experimental observables may provide a test of the consistency between experiments and QCD at higher energies corresponding to the excited states.

The vector and axial-vector current correlators are useful for such analysis because they can be compared to the experimental observable in hadronic $\tau$ decays through the dispersion relation. The early ALEPH data [ [, [ ] ] of the $\tau$ decay experiment were converted to the vector and axial-vector correlators [3] and the result was used to test the consistency with a quenched lattice calculation at a single lattice spacing [团].

With unquenched lattice simulations and updated ALEPH data [5], we perform a more realistic calculation. Numerical simulations are carried out using $2+1$-flavor Möbius domain-wall fermions on $32^{3} \times 64,48^{3} \times 96$ and $64^{3} \times 128$ lattices at the lattice spacings $a=0.080,0.055$ and $0.044 \mathrm{fm}$, respectively. Pion masses on our ensembles are 500, 400, 300 and $230 \mathrm{MeV}$. We show the extrapolation to the physical pion mass and continuum limits agrees with the ALEPH data.

This analysis is done as a part of our studies on current correlators at short and middle distances to investigate what kinds of information of QCD we can extract from them. In these studies, we have determined renormalization factors of quark currents [ [G] and the chiral condensate [ $[\mathbf{D}]$.

\section{Current correlators}

We calculate the vector and axial-vector current correlators

$$
\Pi_{V}(x)=\sum_{\mu}\left\langle V_{\mu}(x) V_{\mu}(0)^{\dagger}\right\rangle, \quad \Pi_{A}(x)=\sum_{\mu}\left\langle A_{\mu}(x) A_{\mu}(0)^{\dagger}\right\rangle-\Pi_{A}^{(0)}(x),
$$

where the vector and axial-vector currents $V(x)$ and $A(x)$ are iso-triplet. Since the spectral functions obtained through hadronic $\tau$ decays are related to the spin- 1 contribution of the correlators, we subtract from the axial-vector channel the spin- 0 part estimated as the contribution of the ground state pion $\Pi_{A}^{(0)}(x) \simeq z_{0} M_{\pi}^{2} K_{1}\left(M_{\pi}|x|\right) / 2 \pi^{2}|x|$, where $K_{1}$ stands for the modified Bessel function and $z_{0}$ and $M_{\pi}$ are extracted from the zero-momentum correlator of the axial-vector current, $\int \mathrm{d}^{3} x\left\langle A_{4}(x) A_{4}(0)^{\dagger}\right\rangle \rightarrow z_{0} \mathrm{e}^{-M_{\pi} x_{4}}$. The vector channel does not need any modification due to the absence of the spin-zero part in the isospin limit. We subtract the discretization effect at the mean field level. The detail is explained in [焑]. 


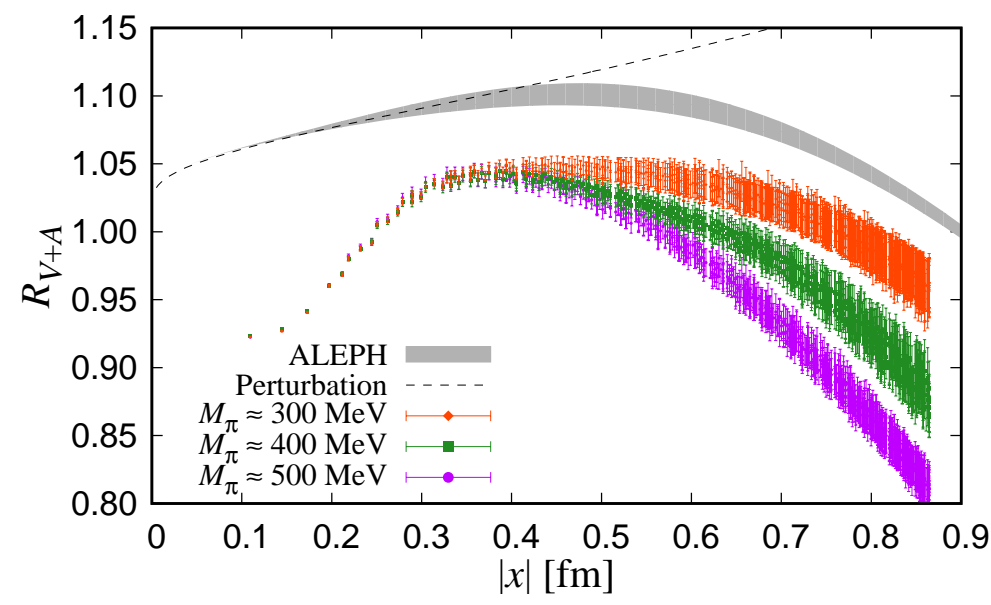

Figure 1: $R_{V+A}$ calculated on the ensembles at $a=0.055 \mathrm{fm}$ and the three pion masses $M_{\pi} \simeq 300 \mathrm{MeV}$ (diamonds), $400 \mathrm{MeV}$ (squares) and $500 \mathrm{MeV}$ (circles). The prediction of massless perturbation theory (dashed curve) and the result from the experiment (band) are also shown.

These correlators are related to the experimentally measured spectral functions $\rho_{V / A}(s)$ through the dispersion relation [目]

$$
\Pi_{V / A}(x)=\frac{3}{8 \pi^{4}} \int_{0}^{\infty} \mathrm{d} s s^{3 / 2} \rho_{V / A}(s) \frac{K_{1}(\sqrt{s}|x|)}{|x|} .
$$

The hadronic $\tau$ decay experiment provides the spectral functions $\rho_{V / A}(s)$ at invariant masses smaller than the $\tau$ lepton mass, $s<m_{\tau}^{2}$. We use the latest ALEPH data $\rho_{V / A}(s)$ of the $\tau$ decay experiment [B] in this region. In the region $s>m_{\tau}^{2}$, we calculate $\rho_{V / A}(s)$ using perturbation theory available to

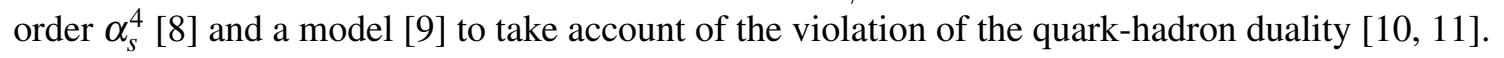

\section{Lattice vs Experiment}

We compare correlators on the lattice with those obtained from the experimental data for the sum and difference $R_{V \pm A}(x)=\left(\Pi_{V}(x) \pm \Pi_{A}(x)\right) / 2 \Pi_{0}(x)$ normalized by the vector correlator in massless free theory $\Pi_{0}(x)$. The $V+A$ channel contains the perturbative contribution, which dominates at short distances. The $V-A$ channel consists only of the effects of the spontaneous chiral symmetry breaking.

First, we focus on the $V+A$ channel. Figure $\square$ shows the results of $R_{V+A}(x)$ calculated on the ensembles at $a=0.055 \mathrm{fm}$ and three different pion masses. The results at smaller masses are closer to the experimental result (band), implying that the chiral extrapolation to the physical pion mass makes them close to the experimental data. Figure $\square$ shows the result at matched pion mass $M_{\pi} \simeq 300 \mathrm{MeV}$ and at different lattice spacings. There is a significant dependence on the lattice spacing, which can be controlled by a term $\propto a^{2}$ at middle and long distances.

We extrapolate these lattice results to the physical point, i.e. the continuum limit $a \rightarrow 0$ and physical pion mass limit $M_{\pi} \rightarrow m_{\pi} \simeq 140 \mathrm{MeV}$ as follows. We divide the range of $|x|$ into $N$ bins,

$$
B_{i}=\left[x_{i}-\delta x, x_{i}+\delta x\right], x_{i+1}=x_{i}+2 \delta x, \quad i=1,2, \ldots, N,
$$




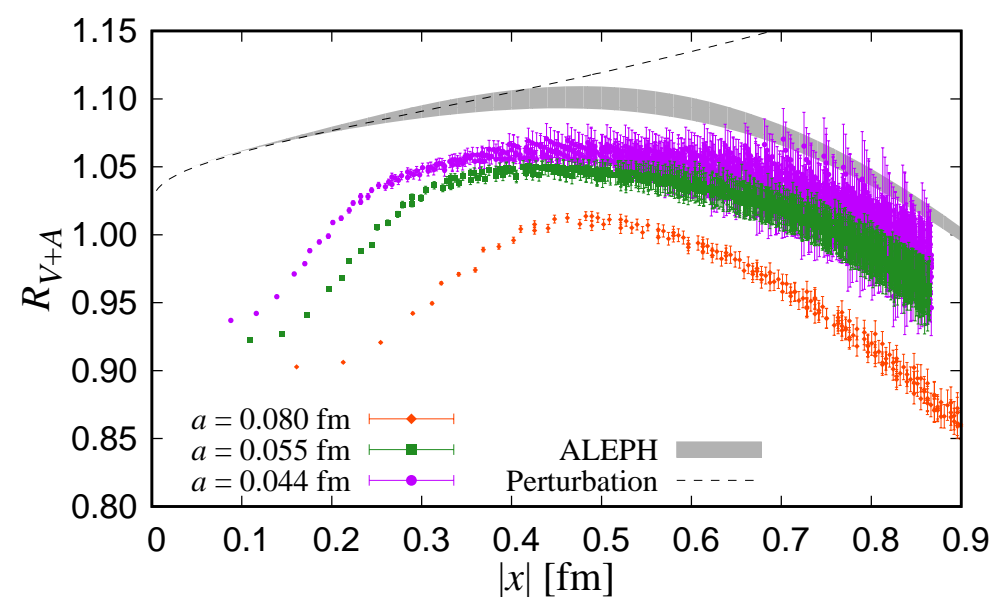

Figure 2: Same as Fig. Wbut calculated on the ensembles at matched pion mass $M_{\pi} \simeq 300 \mathrm{MeV}$ and different lattice spacings $a=0.080 \mathrm{fm}$ (diamonds), $0.055 \mathrm{fm}$ (squares) and $0.044 \mathrm{fm}$ (circles).

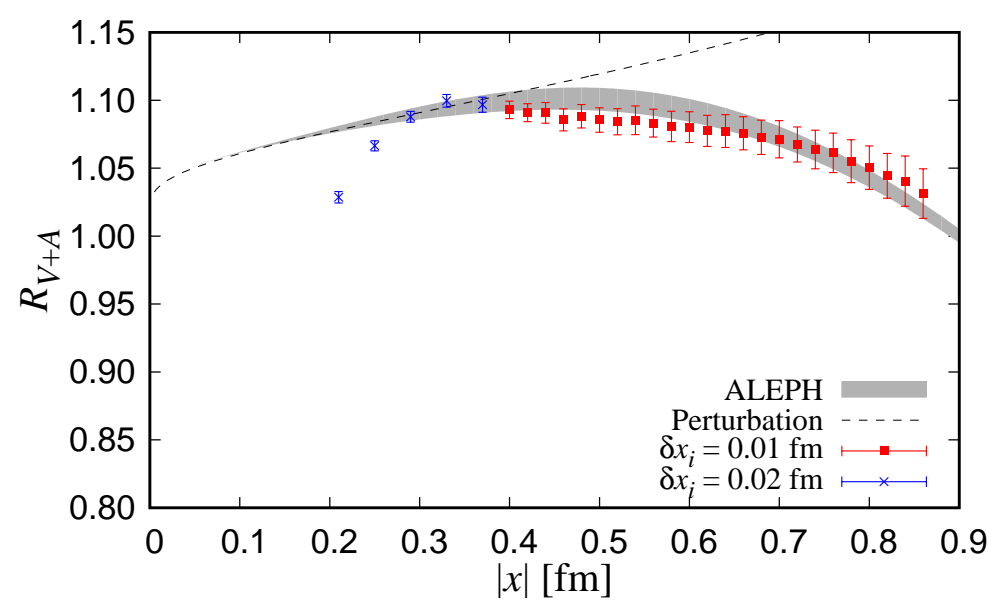

Figure 3: Extrapolation of $R_{V+A}$ to the physical point.

where $x_{i}$ and $\delta x$ are the center of the $i$ th bin and one half of the width of bins, respectively. For each bin, we define $\bar{R}_{V+A}\left(a, M_{\pi}, x_{i}\right)$ as an average of $R_{V+A}(x)$ over the lattice points falling in $B_{i}$. We then perform a global fit for all ensembles using the fit function

$$
\bar{R}_{V+A}\left(a, M_{\pi} ; x_{i}\right)=R_{V+A}\left(0, m_{\pi}, x_{i}\right)+c_{m, i}\left(M_{\pi}^{2}-m_{\pi}^{2}\right)+c_{a, i} a^{2},
$$

with three free parameters $R_{V+A}\left(0, m_{\pi}, x_{i}\right), c_{m, i}$ and $c_{a, i}$ for each $i$. The first parameter $R_{V+A}\left(0, m_{\pi}\right.$, $x_{i}$ ) corresponds to the extrapolated value. The other parameters $c_{m, i}$ and $c_{a, i}$ are to control the dependences on the pion mass and the lattice spacing, respectively. Figure $B]$ shows the result of the extrapolation. Here, we take $\delta x=0.01 \mathrm{fm}$ for $x_{i} \geq 0.4 \mathrm{fm}$ and $\delta x=0.02 \mathrm{fm}$ for $x_{i}<0.4 \mathrm{fm}$. After such an extrapolation, the lattice results are consistent with the experimental data at $|x| \sim 0.3 \mathrm{fm}$ and longer. At even shorter distances, we find deviations from the perturbative and experimental results, which suggest that the discretization effects beyond $O\left(a^{2}\right)$ are significant. In fact, the coefficient $c_{a, i}$ rapidly increases toward the short-distance region. 


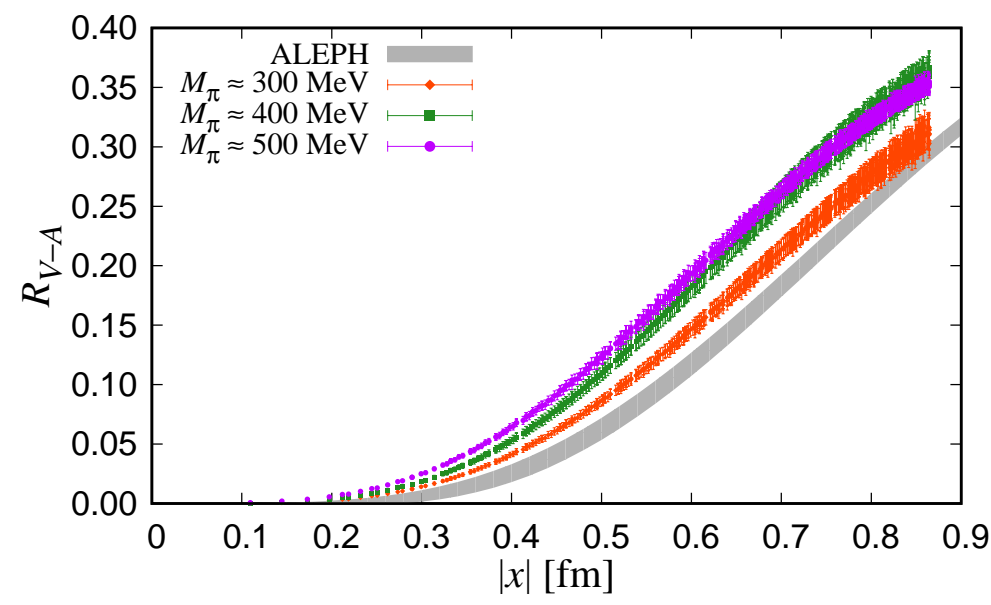

Figure 4: $R_{V-A}$ calculated on the same ensembles as in Fig. W, plotted with the experimental result (band).

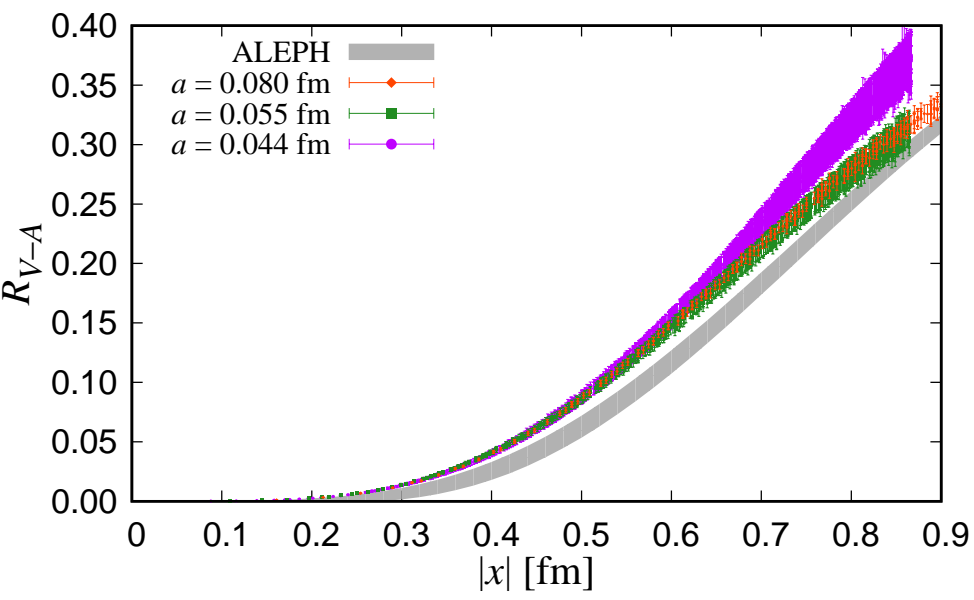

Figure 5: Same as Fig. 团 but calculated on the same ensembles as in Fig. \.

Next, we show the lattice results for the $V-A$ channel. Figure $\mathbb{t}$ shows the lattice data for $R_{V-A}(x)$ at $a=0.055 \mathrm{fm}$ and three different pion masses. $R_{V-A}(x)$ vanishes in the short-distance limit as it should be. Actually it is guaranteed by the good chiral symmetry of Möbius domain-wall fermions. At short distances $(|x| \lesssim 0.5 \mathrm{fm})$, the dependence on the pion mass is clearly seen and the results at smaller masses are closer to the experimental result. In Fig. [1, which shows the data at the matched pion mass $M_{\pi} \simeq 300 \mathrm{MeV}$ and three different lattice spacings, no significant dependence on the lattice spacing is seen at least at short distances unlike the case of the $V+A$ channel. One possible reason for this is that the most of discretization effect on correlators at short distances is perturbative and cancelled for the $V-A$ channel. Figure 6 shows the result of the extrapolation to the physical point, which is done in the same manner as for the $V+A$ channel. A good agreement with the ALEPH data is found.

We also investigate the region of $|x|$ where perturbative approaches give reliable predictions. Figure 6 also shows a rough prediction of the Operator Product Expansion (OPE) [ए]] truncated 


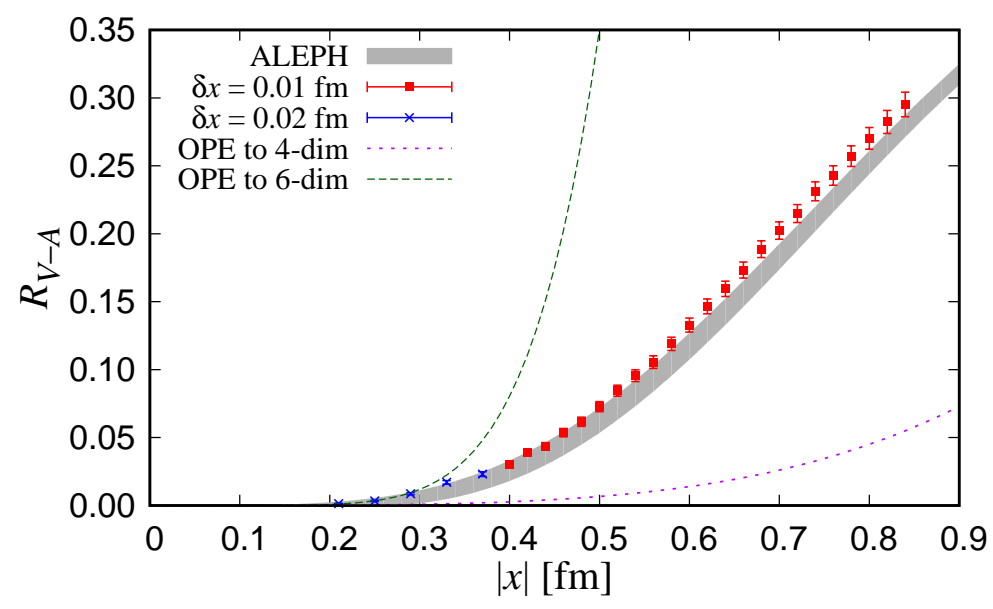

Figure 6: Extrapolation of $R_{V-A}$ to the physical point. The experimental result (band) and the predictions of OPE including up to four- (dotted curve) and six-dimensional (dashed curve) operators are also plotted.

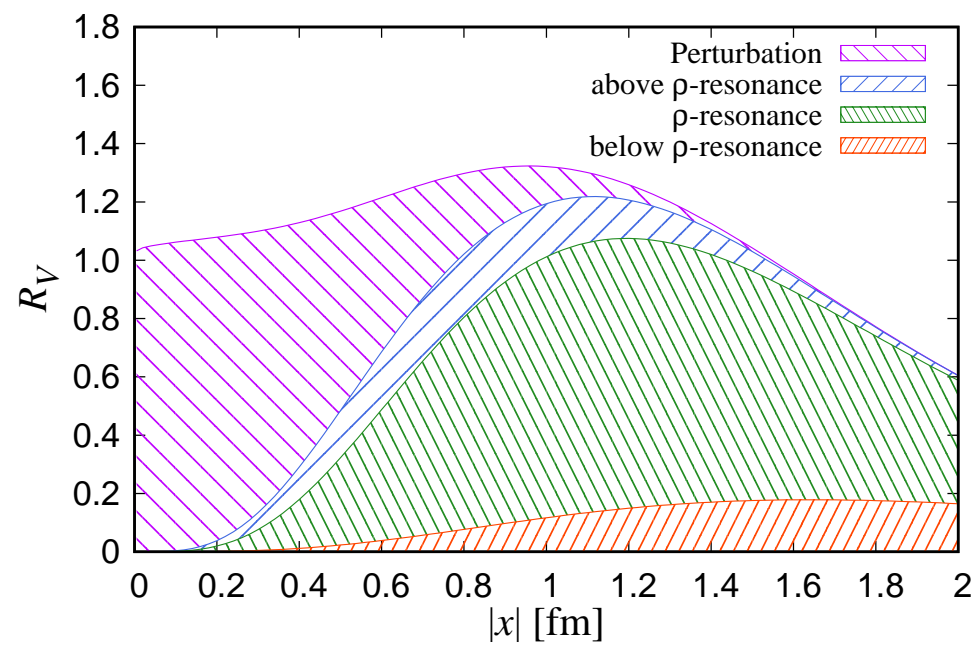

Figure 7: Decomposition of the vector correlators into the contributions of the spectral functions in several regions of $s$. The area indicated by "Perturbation" represents the contribution of the spectral function in $s>2.7 \mathrm{GeV}^{2}$, which is calculated perturbatively. The region $s \leq 2.7 \mathrm{GeV}^{2}$ is splitted into the $\rho$ meson resonance $(0.776-0.150)^{2} \mathrm{GeV}^{2}<s<(0.776+0.150)^{2} \mathrm{GeV}^{2}$ and above and below.

at dimension-four (dotted curve) and dimension-six (dashed curve) operators. The OPE truncated at the dimension-four underestimates the lattice data already at $0.3 \mathrm{fm}$. The OPE including dimension-six operators also disagrees with the lattice result in $|x|>0.3 \mathrm{fm}$. It indicates that the OPE of $R_{V-A}(x)$ is useful in the limited region $|x|<0.3 \mathrm{fm}$. Figure $\square$ shows the decomposition of $R_{V}(x)=\Pi_{V}(x) / \Pi_{0}(x)$ from the experiment into the contributions of the spectral function in several divided regions of $s$. Since the non-perturbative contribution is substantial or even dominant at $|x| \gtrsim 0.5 \mathrm{fm}$, the correlator calculated perturbatively in such a region may not be precise. The axial-vector channel also has a similar decomposition. 


\section{Summary}

We calculate the vector and axial-vector current correlators in the short- and middle-distance regions. We show that the lattice results agree with the ALPEH data of hadronic $\tau$ decays in $|x|>0.3 \mathrm{fm}$ for the $V+A$ channel and $|x|>0.2 \mathrm{fm}$ for the $V-A$ channel.

We also investigate the region where perturbative approaches are useful to describe the correlators. The OPE of the $V-A$ channel disagrees with the lattice result in $|x| \gtrsim 0.3 \mathrm{fm}$. The individual channels of the correlators in $|x| \gtrsim 0.5 \mathrm{fm}$ are not dominated by the contributions from the perturbative regime.

Numerical simulations are performed on Hitachi SR 16000 and IBM System Blue Gene Solution at KEK under a support of its Large Scale Simulation Program (No. 15/16-09, 16/17-14). We thank P. Boyle for the optimized code for BGQ. This work is supported in part by the Grant-inAid of the Japanese Ministry of Education (No. 25800147, 26247043, 26400259) and the Post-K supercomputer project through JICFuS.

\section{References}

[1] R. Barate et al. [ALEPH Collaboration], Z. Phys. C 76, 15 (1997). doi:10.1007/s002880050523

[2] R. Barate et al. [ALEPH Collaboration], Eur. Phys. J. C 4, 409 (1998). doi:10.1007/s100520050217

[3] T. Schäfer and E. V. Shuryak, Phys. Rev. Lett. 86, 3973 (2001) doi:10.1103/PhysRevLett.86.3973 [hep-ph/0010116].

[4] T. A. DeGrand, Phys. Rev. D 64, 094508 (2001) doi:10.1103/PhysRevD.64.094508 [hep-lat/0106001].

[5] M. Davier, A. Höcker, B. Malaescu, C. Z. Yuan and Z. Zhang, Eur. Phys. J. C 74, no. 3, 2803 (2014) doi:10.1140/epjc/s10052-014-2803-9 [arXiv:1312.1501 [hep-ex]].

[6] M. Tomii et al. [JLQCD Collaboration], Phys. Rev. D 94, no. 5, 054504 (2016) doi:10.1103/PhysRevD.94.054504 [arXiv:1604.08702 [hep-lat]].

[7] M. Tomii et al. [JLQCD Collaboration], arXiv:1511.09170 [hep-lat].

[8] P. A. Baikov, K. G. Chetyrkin and J. H. Kuhn, Phys. Rev. Lett. 101, 012002 (2008) doi:10.1103/PhysRevLett.101.012002 [arXiv:0801.1821 [hep-ph]].

[9] O. Cata, M. Golterman and S. Peris, Phys. Rev. D 77, 093006 (2008) doi:10.1103/PhysRevD.77.093006 [arXiv:0803.0246 [hep-ph]].

[10] M. A. Shifman, In Minneapolis 1994, Continuous advances in QCD*249-286 [hep-ph/9405246].

[11] B. Blok, M. A. Shifman and D. X. Zhang, Phys. Rev. D 57, 2691 (1998) Erratum: [Phys. Rev. D 59, 019901 (1999)] doi:10.1103/PhysRevD.57.2691, 10.1103/PhysRevD.59.019901 [hep-ph/9709333].

[12] M. A. Shifman, A. I. Vainshtein and V. I. Zakharov, Nucl. Phys. B 147, 385 (1979). doi:10.1016/0550-3213(79)90022-1 